TECHNICAL NOTE

K. Wang

Q. Huang

B. Hong

Y. Xu

W. Zhao

J. Chen

R. Zhao

J. Liu

\title{
Neck Injury Is Critical to Elastase-Induced Aneurysm Model
}

SUMMARY: We modified the elastase-induced aneurysm model by use of a simple surgical technique in rabbits. A temporary arcuated aneurysm clip was placed at the origin of the right common carotid artery (RCCA), ascertaining the inner edge of the clip blade on the juncture of the RCCA and right subclavian artery (RSCA), and the elastase-induced aneurysm procedure was undertaken. We found elastase and location of the temporary arcuated aneurysm clip are critical to the success of this aneurysm model.

$\mathbf{T}$ he elastase-induced aneurysm model has been gradually accepted in recent years because it resembles a human aneurysm in hemodynamics and histology. ${ }^{1,2}$ The creation of this model requires a digital subtraction angiography (DSA) machine and a disposable occlusion balloon. Moreover, inflation of the balloon at the origin of the right common carotid artery (RCCA) may also injure the intima of the brachiocephalic artery and the origin of the RCCA. The aberrant origins of the branch to the trachea from the RCCA are areas through which elastase could pass and lethal hemorrhagic necrosis of the trachea could occur. ${ }^{3}$ We used a simple modified surgical technique to create an elastase-induced aneurysm model and observed the stability and pathologic changes of this model in rabbits.

\section{Technique}

A total of 33 New Zealand white rabbits were randomly divided into 3 groups: group $1(n=23)$, group $2(n=5)$, and group $3(n=5)$.

In group 1 , by using sterile techniques, we made a middle skin incision extending from the thyroid cartilage to the manubrium sterni. Working between the right sternomastoid muscle and trachea, we carefully exposed the RCCA. Two 3-0 silk ligatures were twisted to mark the RCCA. Then soft tissue was dissected to the right of the sternomastoid muscle until the 2 silk ligature marks were found. The RCCA was exposed, guided by the silk ligatures. A part of the right pectorals was dissected. The origin of the RCCA and part of right subclavian artery (RSCA) were then dissected carefully. The RCCA was ligatured $2.5 \mathrm{~cm}$ distally from the origin. A temporary arcuate aneurysm clip was placed at the origin of RCCA, indicating the inner edge of clip blade on the juncture of the RCCA and RSCA (Fig 1). The RCCA was ligated by a lax single-tied silk suture (size 1), $5 \mathrm{~cm}$ prox-

Received October 27, 2008; accepted after revision January 11, 2009

From the Department of Neurosurgery, Changhai Hospital of Second Military Medical University, Shanghai, P.R. China.

This work was supported by the Program of Shanghai Subject Chief Scientist (07XD14026) and sponsored by the Science and Technology Commission of Shanghai Municipality.

Please address correspondence to Jianmin Liu, MD, Department of Neurosurgery, Changhai Hospital of Second Military Medical University, Changhai Rd 168, Shanghai 200433, P.R. China; e-mail: liujm8@163.com

Indicates open access to non-subscribers at www.ajnr.org

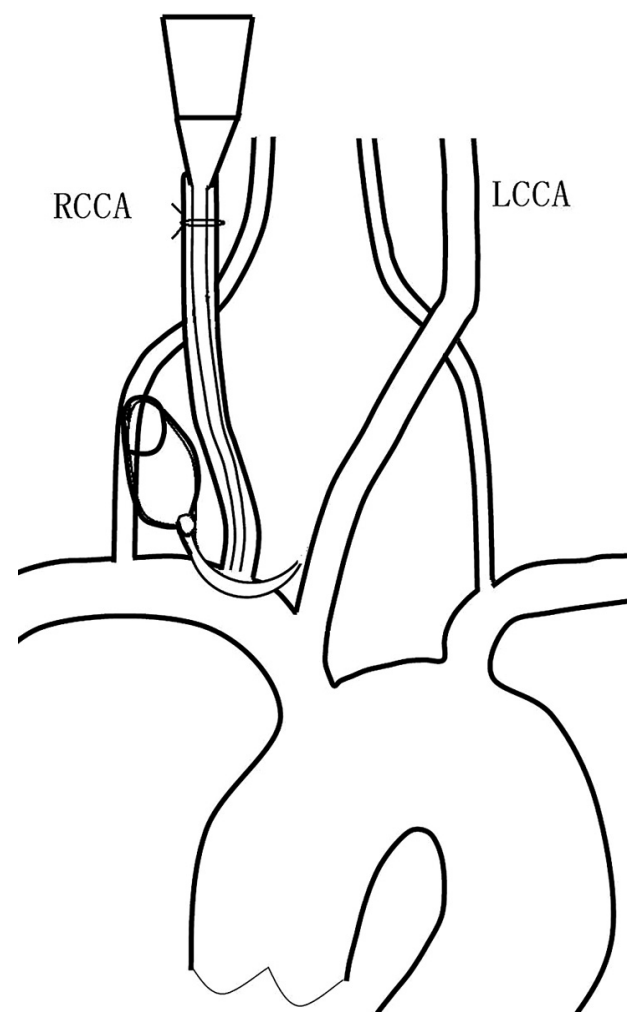

Fig 1. Schematic of elastase-induced aneurysms in rabbits in groups 1 and 2. A temporary arcuate aneurysm clip is placed at the origin of the RCCA, indicating the inner edge of clip blade on the juncture of RCCA and right subclavian artery. LCCA indicates left common carotid artery.

imal to the origin. A 1-mm beveled arteriotomy was made beside the planned suture site, and a 22-gauge integrated catheter was advanced retrograde to the origin of RCCA. The lax suture was fastened. A total of 75-U porcine pancreatic elastase was incubated for 20 minutes within the vessel lumen. The integrated catheter was removed, and the suture at the integrated catheter site was ligated at the same time; then the temporary arcuate aneurysm clip was released. Heparin saline (200 U/kg) was administered intravenously, and penicillin was injected intramuscularly. Muscle and skin were closed separately with 2-0 sutures. The animal was allowed to recover.

The animals in group 2 underwent the same procedure as those in group 1, except that elastase was replaced by saline. The animals in 

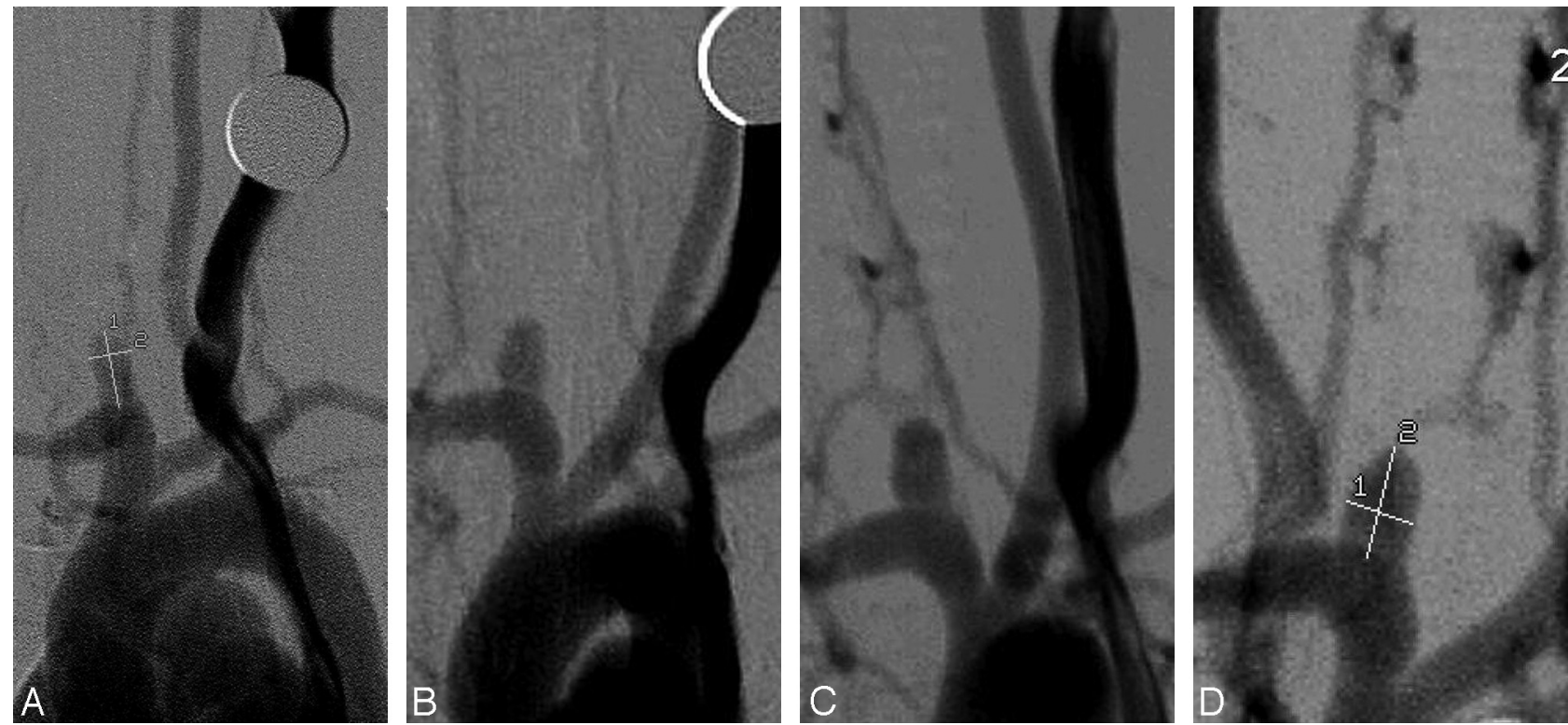

Fig 2. Intravenous digital subtraction angiography in a rabbit from group 1. The aneurysm is stable at 3 weeks, 8 weeks, 4 months, and 6 months after surgery.
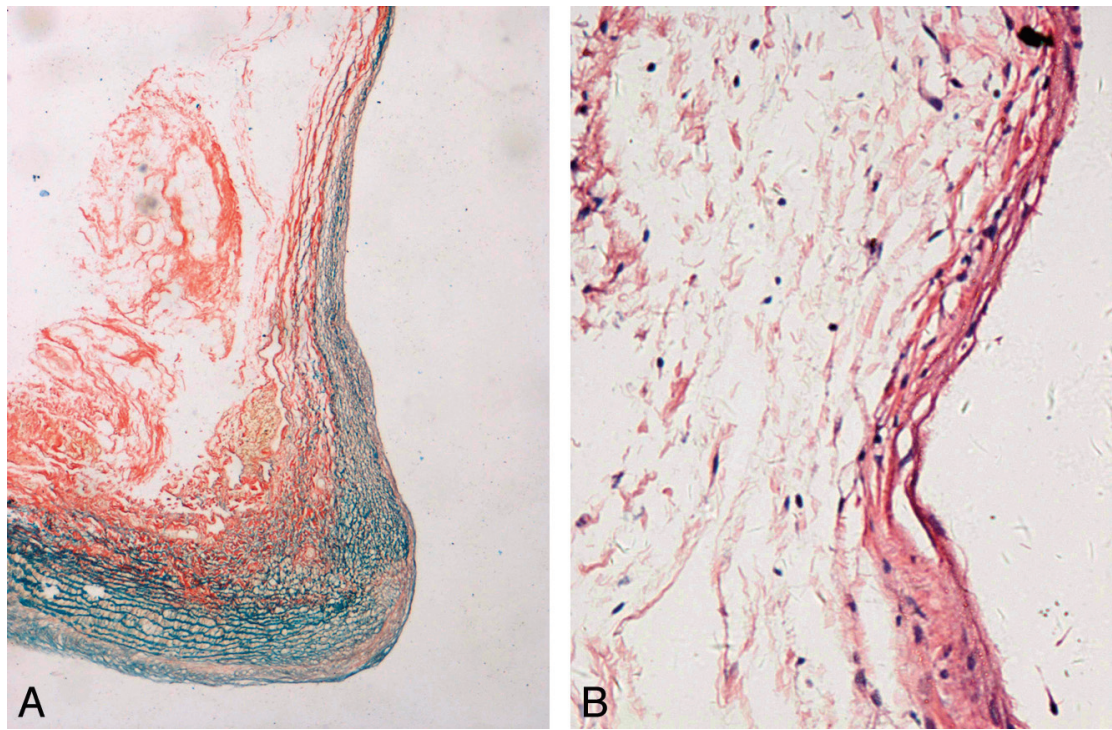

Fig 3. Histopathologic analysis of the right common carotid artery (RCCA) in group 1. $A$, The RCCA induced by elastase after 3 weeks, stained by ponceau/Victoria blue, becomes an aneurysm with intact elastin in the parent vessel and abrupt termination of the elastic lamina at the margins of aneurysm orifice (original magnification $\times 40$ ). $B$, Hematoxylin-eosin staining indicates a thin aneurysm wall, the absence of an inflammatory reaction, and marked loss of cellular elements (original magnification $\times 200$ ).

group 3 also underwent the same procedure as those in group 1, except that the temporary arcuate aneurysm clip location was $3 \mathrm{~mm}$ away from the RCCA origin.

Intravenous DSA (IVDSA) was performed at each time point (3 weeks, 8 weeks, 4 months, and 6 months after the procedure). Two animals from group 1 and 1 animal from each of the other groups were euthanized after angiography at each time point.

In group 1, 20 of 23 rabbits that underwent the aneurysm-creation procedures showed saccular aneurysms on IVDSA 3 weeks after the procedure ( $87 \%$ successful outcome), whereas the other 3 animals showed a residual dog-ear remnant at the RCCA stump with a diameter of $<2 \mathrm{~mm}$. There were no significant differences in the mean sizes of the aneurysms in width, height, and neck or in the mean parentvessel diameter at 3 and 8 weeks, and 4 and 6 months after the procedure (Fig 2). In groups 2 and 3, all animals showed residual dog-ear remnants at the RCCA stump with a diameter of $<2 \mathrm{~mm}$ at every observation point.

In group 1, the stump of the RCCA was aneurysmally dilated and transparent, and thrombus in the dome was observed at week 3 (Fig 3 ). The aneurysmal dilation was opaque at week 8 and at months 4 and 6, the thrombus in the dome had been absorbed, and the saccular wall had partly thickened due to neointimal or adventitia proliferation. In group 2, the residual of the RCCA was not dilated and showed normal arterial structure from any viewpoint. In group 3, the stump of the RCCA was slightly dilated but was full of thrombus on gross pathology at week 3. Pathology showed abrupt termination of elastic layers and no elastin $3 \mathrm{~mm}$ from origin of the RCCA. At week 8 and at months 4 and 6, the RCCA 3-mm distance from origin became a linelike residual and showed less cell tissue, but the origin of the RCCA was normal. 


\section{Discussion}

Hoh et $\mathrm{al}^{4}$ reported a new modified technique to the elastase aneurysm model, in which the rabbit RCCA origin was surgically exposed and temporarily occluded with an aneurysm clip and the tapped segment was infused with elastase. However, surgery of the sternothyroid muscle, pectoralis tenuis muscle, RCCA, RSCA, and brachiocephalic trunk may injure the animal excessively, and the straight temporary aneurysm clip occupies much surgical space and is not convenient in practice. We, thus, created an elastase aneurysm model by modifying the method of Hoh et al. ${ }^{4}$

Our method has 3 advantages: First, we used a temporary arcuate aneurysm clip, which occupied less surgical space and was convenient in practice, to occlude the origin of the RCCA. Second, the RSCA was partly dissected, and the brachiocephalic trunk was untouched, causing less injury to the animal. Third, the arc of the temporary arcuate aneurysm clip partly protruded into the RSCA, and the tip of the integrated catheter approached the clip, which ensured that the neck of aneurysm was digested sufficiently by elastase. Using the position of the aneurysm clip described by Hoh et $\mathrm{al}^{4}{ }^{4}$ we found that it was difficult to induce an aneurysm or only an aneurysm with a narrow neck in our preliminary experiments. We think the reason for this was that the origin of the RCCA remained 1 $\mathrm{mm}$, undigested by elastase (the width of aneurysm clip blade was approximately $1 \mathrm{~mm}$ ) and thus did not cause the hemodynamics of the aneurysm to be significantly changed.

Our results also indicated that elastase and the location of the temporary aneurysm clip are important in the formation of aneurysm models. Ding et $\mathrm{al}^{5}$ reported that the neck size of an elastase-induced aneurysm could be controlled by adjusting the position of the occlusion balloon during elastase incu- bation and that a higher position of the balloon induced narrow necks and higher dome-to-neck-ratio aneurysms. Thiex et $\mathrm{al}^{6}$ confirmed that balloon occlusion in the brachiocephalic trunk resulted in broad-based aneurysms, whereas balloon occlusion in the RCCA gave rise to circumscribed aneurysm necks. The rabbits in group 3 of our study did not show aneurysms on IVDSA. We think that this was secondary to the low flow rate in the aneurysm, because the temporary aneurysm clip, which was placed $3 \mathrm{~mm}$ from the RCCA origin, protected the neck region from elastase injury. Therefore, mere surgical creation of an RCCA stump without elastase did not produce an aneurysm in group 2, and animals in group 3 showed the fully filled thrombosis instead of aneurysm formation because of the lesion in the vascular wall and low hemodynamic stresses.

\section{References}

1. Ding YH, Dai D, Lewis DA, et al. Long-term patency of elastase-induced aneurysm model in rabbits. AJNR Am J Neuroradiol 2006;27:139-41

2. Krings T, Busch C, Sellhaus B, et al. Long-term histological and scanning electron microscopy results of endovascular and operative treatments of experimentally induced aneurysms in the rabbit. Neurosurgery 2006;59:911-23, discussion 923-24

3. Thiex R, Hans FJ, Krings T, et al. Haemorrhagic tracheal necrosis as a lethal complication of an aneurysm model in rabbits via endoluminal incubation with elastase. Acta Neurochir (Wien) 2004;146:285-89, discussion 289. Epub 2004 Jan 22

4. Hoh BL, Rabinov JD, Pryor JC, et al. A modified technique for using elastase to create saccular aneurysms in animals that histologically and hemodynamically resemble aneurysms in human. Acta Neurochir (Wien) 2004;146:705-11

5. Ding YH, Dai D, Lewis DA, et al. Can neck size in elastase-induced aneurysms be controlled? A prospective study. AJNR Am J Neuroradiol 2005;26:2364-67

6. Thiex R, Möller-Hartmann W, Hans FJ, et al. Are the configuration and neck morphology of experimental aneurysms predictable? A technical approach. Neuroradiology 2004;46:571-76 